\title{
L'envasement dans différents ports de Loire-Atlantique
}

\author{
Martin Sanchez ${ }^{1}$, et Yann Delanoë ${ }^{1}$ \\ ${ }^{1}$ Université de Nantes, Faculté des Sciences et des Techniques, \\ UMR-CNRS 6112 Laboratoire de Planétologie et Géodynamique, \\ 2 rue de la Houssinière, BP 92208, 44322 Nantes \\ martin.sanchez@univ-nantes.fr ; yann.delanoe@univ-nantes.fr
}

\begin{abstract}
Résumé
Les distributions des dépôts de vase et des turbidités des eaux dans l'estuaire de la Loire et dans la région littorale de Loire-Atlantique sont étudiées. La dynamique sédimentaire dans ces deux régions est décrite en relation avec les courants de marée et avec l'agitation liée à la houle et aux clapots.

Le problème d'envasement est examiné dans plusieurs sites portuaires en faisant le lien avec les conditions environnantes comme la courantologie et la turbidité des eaux, et avec l'hydrodynamique locale associée à la configuration des ouvrages maritimes. Enfin, tenant compte des coûts de dragages et des conditions environnantes, des configurations sont suggérées pour l'implantation des ports de pêche et de plaisance en Loire-Atlantique.
\end{abstract}

\begin{abstract}
The distributions of mud deposits and turbidities are studied in the estuary of the river Loire and in the coastal area of Loire-Atlantique (France). A description of the sediment dynamics is given in regard to tidal streams and water agitation due to waves.

The silting up of the harbour sites is investigated in relation with the environmental conditions and local hydrodynamics associated with the harbour infrastructures.

Some configurations are suggested for the implantation of fishing ports and marinas, in Loire-Atlantique, taking into account economical and environmental conditions.
\end{abstract}

Mots clés : vase, envasement, dragage, dépôt, port, estuaire, Loire-Atlantique.

Key-words: mud, silting up, dredging, deposits, harbour, estuary, Loire, France.

\section{$\underline{\text { 1. Introduction }}$}

Les sédiments cohésifs de type vase s'accumulent préférentiellement dans certains endroits du littoral. Les principaux sites affectés par l'envasement sont : les zones marécageuses, les estuaires, les régions côtières protégées de l'action de la houle et certaines zones océaniques du plateau continental. 
Dans la plupart des cas l'envasement est ressenti comme une entrave à la navigation ou une nuisance environnementale car les vases sont susceptibles de piéger les matières organiques, les métaux lourds, les pollutions chimiques, et peuvent avoir une mauvaise qualité bactérienne, ce qui les rend souvent inadaptées à la construction, à l'agriculture, et à toute autre valorisation.

Ainsi, l'envasement implique souvent la mise en œuvre de travaux de dragage onéreux et peu efficaces du fait de la très forte teneur en eau. Contrairement aux sables dragués qui sont fortement revalorisés, les vases ne le sont que très peu dans le rechargement de vasières et la construction de remblais. La plus grande partie des vases draguées est rejetée en mer en eaux profondes, ou confinée à terre, occupant des sites sans aucune autre utilisation quand les matériaux sont trop pollués.

L'envasement dans un bassin portuaire dépend d'une part des conditions environnantes comme la courantologie et la turbidité des eaux, et d'autre part de l'hydrodynamique interne associée à la configuration des ouvrages maritimes.

Le but de cette étude n'est pas la modélisation de l'ensemble des processus hydrosédimentaires affectant la dynamique des vases, mais plutôt l'évaluation des risques d'envasement, tenant compte des données disponibles. Les évolutions morphologiques à très long terme ne sont pas particulièrement abordées. On s'intéresse surtout aux problèmes d'envasement observés sur des périodes allant de quelques jours à quelques années.

\section{Les conditions environnementales}

La dynamique sédimentaire des matériaux cohésifs dépend d'un certain nombre de conditions environnementales parmi lesquelles les plus importantes sont la distribution des dépôts de vase, les turbidités des eaux, et les conditions hydrodynamiques. Ces conditions sont décrites par la suite pour les régions étudiées dans cette note.

\subsection{La dynamique des vases dans l'estuaire de la Loire}

Les sédiments rencontrés dans les fonds de l'estuaire de la Loire sont en étroite relation avec les conditions hydrauliques régnant dans chaque secteur. Ainsi, on constate que la vase est le sédiment prédominant dans le chenal de l'estuaire en aval de Nantes (situé à $55 \mathrm{~km}$ de l'embouchure, Figure 1), tandis qu'en amont de ce site on trouve principalement du sable.

L'estuaire de la Loire comporte une zone de bouchon vaseux (turbidité maximale) très marquée où la concentration $C$ en matières en suspension (MES) peut être de 10 à 200 fois celle observée dans le fleuve dans des conditions moyennes, qui est de l'ordre de $0,06 \mathrm{~kg} \mathrm{~m}^{-3}$. La zone du bouchon vaseux a une étendue moyenne de $20 \mathrm{~km}$, et son centre de gravité se déplace en fonction du débit fluvial (Migniot et Le Hir, 1994).

En mortes-eaux, une grande partie des matières en suspension s'accumulent au fond pour former une lentille de vase fluide ou crème de vase. Cette formation peut atteindre $12500 \mathrm{~m}$ de longueur par $300 \mathrm{~m}$ de largeur et par $2 \mathrm{~m}$ d'épaisseur. 
La concentration en matières sèches de la crème de vase varie entre $40 \mathrm{~kg} \mathrm{~m}^{-3}$ et $325 \mathrm{~kg} \mathrm{~m}^{-3}$, avec un gradient vertical qui est fonction du temps de tassement.

En vives-eaux (coefficient de marée supérieur à 75 dans l'estuaire de la Loire), la plus grande partie de la crème de vase est remise en suspension (Gallenne, 1974), ne restant au fond que les sédiments ayant dépassé une concentration voisine de $300 \mathrm{~kg} \mathrm{~m}^{-3}$, ce qui correspond à environ une semaine de tassement. Ces sédiments, qui résistent sans érosion significative aux courants extrêmes de viveseaux, contribuent à l'engraissement résiduel de l'estuaire.

Les sédiments fins qui se trouvent alternativement en suspension en viveseaux, et sous la forme de crème de vase en mortes-eaux, constituent un stock de vase "mobile". Dans l'estuaire de la Loire ce stock représente en moyenne environ $1 \times 10^{9} \mathrm{~kg}$ de sédiments (CSEEL, 1984 ; Delft hydraulics, 1994 ; Fera, 1994), soit l'équivalent de 8 mois d'apport par le fleuve dans des conditions normales.

Dans l'estuaire de la Loire, le stock de vase "mobile" est situé à l'embouchure en crue et en amont de la ville de Nantes lors d'étiages sévères, au voisinage de la limite supérieure de l'intrusion saline. La présence de ce stock s'explique d'une part, par le dépôt des matières en suspension qui se produit dans ce secteur de l'estuaire pendant la renverse des courants, et d'autre part, par les courants résiduels près du fond, lesquels convergent vers un point nodal situé dans ce secteur.

\subsection{La dynamique des vases devant les côtes de Loire-Atlantique}

Les sédiments déposés à proximité des côtes de Loire-Atlantique ont été étudiés par plusieurs auteurs : Papy (1941), Gouleau (1975), LCHF (1987), CMB Loire Estuaire (2003). Les cartographies des sédiments superficiels dans le secteur présentent des différences entre elles, dues en partie à l'échelle des représentations. Mises à part ces différences, l'ensemble d'études fait ressortir 4 zones d'envasement en face des côtes de Loire-Atlantique. Ces zones contiennent une grande quantité de vase consolidée "peu mobile" ne participant que très peu à la dynamique sédimentaire. La masse de ce stock n'a pas été estimée mais elle est considérable et représente certainement l'équivalent d'un grand nombre de siècles d'apports solides en suspension en provenance des eaux continentales. Les quatre zones d'envasement (Figure 1) sont :

Zone 1.- Baie de Bourgneuf. Les sédiments fins déposés dans ce secteur proviennent principalement des anciennes expulsions de l'estuaire de la Loire. L'envasement s'explique par une agitation très faible due à la présence de l'île de Noirmoutier. Les vases couvrent la plus grande partie de cette baie à l'exception de la partie haute des estrans où les sables prédominent. On y trouve également un très important stock de crème de vase facilement mobilisable par les vagues du fait des profondeurs relativement faibles dans ce secteur.

Zone 2.- Estuaire externe de la Loire. Les sédiments dans ce secteur sont en relation directe avec l'estuaire de la Loire. Cette zone située au fond de la grande rade de Loire est relativement bien abritée de l'action de la houle, bien que l'agitation y soit supérieure à celle observée en baie de Bourgneuf. Certaines études envisagent la possibilité que les vases stockées dans ce site puissent contribuer dans les conditions hydrologiques actuelles à l'envasement de l'estuaire 
interne de la Loire (Delft hydraulics, 1994, DHI, 2003). Cependant, d'autres auteurs envisagent sur le long terme un débit net solide de l'estuaire interne vers l'estuaire externe de l'ordre de $0,5 \times 10^{9} \mathrm{~kg} \mathrm{an}^{-1}$ (Migniot, 1993). Un stock de crème de vase mobilisable est également présent dans ce secteur.

Zone 3.- Zone de la Lambarde vers l'ouest. Ce secteur est assez exposé à l'action de la houle en provenance du large. L'envasement de ce site ne peut donc se produire que les jours d'eau calme, il s'explique par l'arrivée des vases en suspension évacuées de la Loire par les courants de jusant. Le taux d'envasement naturel de ce secteur est certainement plus faible que ceux observés en baie de Bourgneuf et dans l'estuaire externe de la Loire. A cet envasement naturel on doit ajouter un envasement d'origine anthropique lié au déversement par clapage des vases draguées dans le chenal de navigation de la Loire. Les dragages d'entretien clapés dans ce secteur entre 1980 et 1992 ont représenté l'équivalent de $34 \times 10^{6}$ $\mathrm{m}^{3}$ de matériaux (vase et sable) de densité ramenée à 1,40 (Migniot, 1993), soit environ $22 \times 10^{9} \mathrm{~kg}$ de matériaux, quantité sensiblement égale à celle apportée par charriage et en suspension par le fleuve sur la même période. Dans la période 1993-2001, les sédiments dragués et transportés ont été évalués à environ $6 \times 10^{6}$ $\mathrm{m}^{3} \mathrm{an}^{-1}$ (DHI, 2003). Les profondeurs étant importantes (supérieures à $18 \mathrm{~m}$ ) il est peu probable que les vases déposées puissent être remises en suspension, sauf en cas de très grosse houle.

Zone 4.- Baie de la Vilaine. Toute la baie est envasée du fait d'une forte atténuation de la houle dans ce secteur. Les dépôts de vase se prolongent au sud jusqu'au devant des côtes situées à l'ouest du Croisic. Il est vraisemblable que ces vases soient d'origine marine en relation avec les anciennes expulsions de la Vilaine mais aussi de la Loire. La construction du barrage d'Arzal en 1970 a modifié les conditions hydrauliques surtout juste à l'aval du barrage (LCHF, 1987), provoquant depuis un fort envasement dans ce secteur. Dans cette baie on trouve aussi un très important stock de crème de vase facilement mobilisable en cas de tempête.

On doit noter que les stocks de crème vase mobilisables jouent un rôle majeur sur les taux d'envasement observés dans les ports. En effet, en cas de forte agitation provoquée par les tempêtes, la crème de vase est remise en suspension, faisant augmenter considérablement les concentrations en matières en suspension dans les eaux entrant dans les ports pendant le flot.

La figure 2 (Castaing, 1981) montre la distribution des turbidités des eaux superficielles estimée à partir de la mesure de la réflectance de la surface océanique sur des images satellite. L'image a été enregistrée le 8 mars 1973 en vives-eaux, par un débit fluvial moyen de $930 \mathrm{~m}^{3} \mathrm{~s}^{-1}$.

Aucune échelle quantitative n'est indiquée pour les valeurs de la concentration. Les plus fortes turbidités sont observées dans les estuaires interne et externe de la Loire et dans la baie de Bourgneuf. Ces turbidités pourraient dépasser $1 \mathrm{~kg} \mathrm{~m}^{-3}$. Les turbidités les plus faibles observées sur la bande côtière sont celles régnant au nord du Croisic ; elles seraient du même ordre de grandeur que les turbidités observées au large, c'est-à-dire, inférieures à $0,005 \mathrm{~kg} \mathrm{~m}^{-3}$. 


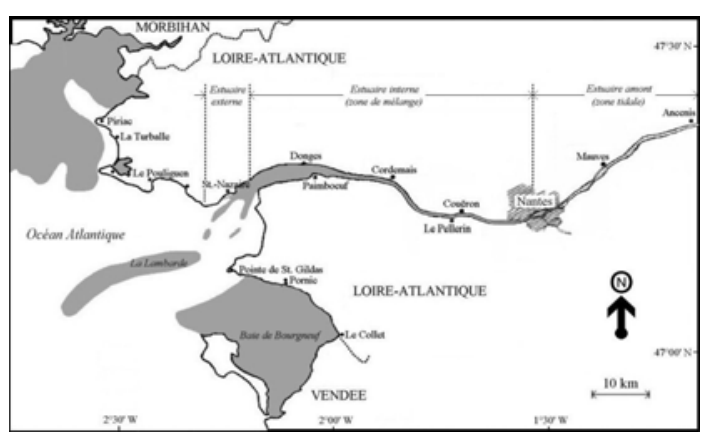

Figure 1 : Dépôts superficiels de vase devant les côtes de Loire-Atlantique et dans l'estuaire de la Loire (carte élaborée à partir de données publiées par LCHF (1987) et CMB Loire Estuaire (2003)).

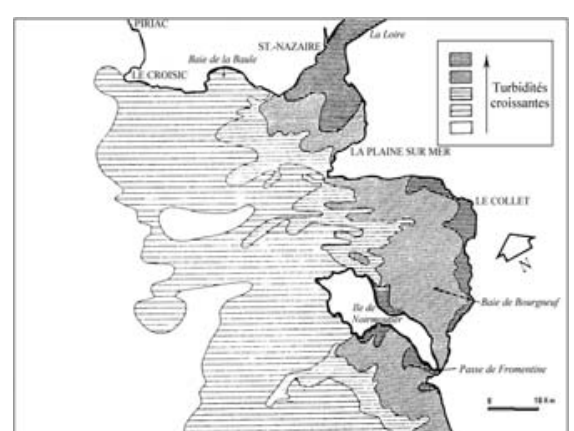

Figure 2 : Turbidités à l'embouchure de la Loire et en Baie de Bourgneuf le 8 mars 1973 par un débit fluvial de la Loire de $930 \mathrm{~m}^{3} \mathrm{~s}^{-1}$ (Castaing, 1981).

Un plus important nombre d'images satellites a été examiné pour l'étude des turbidités dans ce secteur (Froidefond et al., 2003). Même s'il apparaît que les turbidités sont très variables suivant les conditions de débit fluvial, de coefficient de marée et d'agitation de l'eau, la figure 2 reste représentative de la répartition des turbidités maximales.

Le fait que les matières en suspension observées dans les zones de forte turbidité de la baie de Bourgneuf et au sud de l'île de Noirmoutier ne soient pas expulsées vers le large est assez difficile à expliquer, car la présence d'un point nodal comme celui observé au fond des estuaires est peu plausible dans ce secteur.

Un mécanisme faisant intervenir la floculation et la défloculation de matières en suspension a été suggéré pour expliquer le maintien d'une zone de turbidité relativement élevée (concentrations de l'ordre de $0,010 \mathrm{~kg} \mathrm{~m}^{-3}$ ) en mer d'Irlande (Bowers et al., 2005). Il semble peu probable que ce mécanisme s'applique ici.

\section{Le chenal de navigation de l'estuaire de la Loire}

La tradition attribue à Saint Félix la création du port de Nantes au VI ${ }^{\text {ème }}$ siècle. Il aurait fait creuser le canal qui porte son nom et un chenal qui, après avoir reçu l'étier de Mauves, aboutissait au petit port de Richebourg (Denizeau, 1988). Il semble que déjà à cette époque l'envasement et l'ensablement de ce port entravaient la navigation. C'est en grand partie à cause des problèmes sédimentaires que le port glisse de sa situation initiale vers l'ouest : de Richebourg à Briand Maillard au XIII ${ }^{\text {ème }}$ siècle, puis à La Fosse au XVI ${ }^{\text {ème }}$ siècle.

L'aménagement de l'estuaire de la Loire depuis la seconde moitié du XIX ${ }^{\text {ème }}$ siècle a eu, comme l'un de ses principaux objectifs, l'entretien d'une voie navigable d'accès au port de Nantes. L'ouverture du canal de la Martinière, long de $15 \mathrm{~km}$, en 1892, permettait de franchir le secteur s'étendant entre Paimboeuf et Le Pellerin, où les nombreux chenaux entourant les îles étaient trop instables pour la navigation. Même si ce canal a donné satisfaction, son abandon s'explique par un tirant d'eau ne pouvant pas dépasser 5,80 m. 
Par la suite, après 1903, les travaux d'aménagement inspirés par l'ingénieur Léchalas visaient à utiliser l'énergie des courants pour entretenir les fonds : le volume d'eau emmagasiné en flot dans le bassin de marée situé dans la partie amont de l'estuaire devait entretenir, pendant le jusant, des profondeurs suffisantes dans la voie de navigation. Léchalas a également préconisé d'établir un chenal unique, de tracé régulier entre Paimboeuf et Le Pellerin, et une rectification du lit du fleuve afin de faciliter la remontée de la marée. Les travaux ont comporté des dragages d'approfondissement et la construction de digues et d'épis.

Suite à ces grands travaux d'aménagement, le chenal d'accès au port de Nantes permet à l'heure actuelle un tirant d'eau de l'ordre de $6 \mathrm{~m}$ à basse mer de vive-eau exceptionnelle, avec très peu de dragages d'entretien. De même, l'augmentation de la capacité d'évacuation de crues aurait fait diminuer les risques d'inondation à Nantes. En revanche, trois conséquences néfastes ont été constatées :

a) un abaissement des basses mers qui met en danger la stabilité des anciens quais de la ville de Nantes et les ouvrages de protection des berges de l'estuaire et provoque aussi un assèchement des zones humides,

b) une remontée du front de salinité à $0,5 \%$ en vives-eaux et en étiage, qui se situait au Pellerin (PK 38) en 1948 et atteint le PK 70 en 1991 en étiage,

c) une remontée de la zone de forte turbidité des eaux (bouchon vaseux) qui se situe aux environs du front de salinité.

Ces modifications sont également en relation avec un surcreusement du bassin de marée par suite de l'extraction d'environ $75 \times 10^{6} \mathrm{~m}^{3}$ de sable en amont de Nantes entre 1910 et 1990 (Migniot, 1993).

Le développement portuaire à proximité de l'embouchure de l'estuaire s'est matérialisé depuis 1933. Les installations portuaires pour les plus grands navires sont actuellement celles de Montoir et de Donges qui, depuis 1980, permettent l'accueil à basse mer de bateaux avec un tirant d'eau de l'ordre de 13,50 m.

Dans les conditions actuelles, l'essentiel des travaux de dragage d'entretien concernent le chenal de l'estuaire en aval de Donges, alors que l'entretien du chenal entre Nantes et Donges ne représente qu'environ 12\% du total.

Afin de tenter d'expliquer le fort envasement observé en aval de Donges, nous appliquons avec prudence une méthode issue des études de calibrage et de stabilité des passes reliant la mer et les lagunes littorales. En effet, par l'observation de passes relativement stables, plusieurs formules empiriques ont été ajustées pour relier la section transversale moyenne au volume d'eau oscillant (Bruun, 1978).

Même si chacune de ces formules n'est valide que dans une région géographique restreinte, Jarrett (1976) a ajusté une loi de tendance (trait continu de la figure 3) valide en moyenne pour toutes les régions étudiées. On constate sur la figure 3 que les points calculés pour différents secteurs dans l'estuaire entre Nantes et l'embouchure, correspondent à des sections transversales stables. Les surfaces des sections transversales seraient donc bien calibrées.

En revanche les profondeurs en aval de Donges semblent être trop importantes pour permettre que les courants de vive-eau remettent en suspension les couches de crème de vase déposées en morte-eau. La stabilité des fonds peut être étudiée à travers la tension de cisaillement au fond $\tau_{o}$, car les théories hydrodynamiques actuelles fixent les conditions de début d'érosion en fonction de ce paramètre. 
La figure 4 montre plusieurs valeurs de $\tau_{o}$ calculés pour l'estuaire de la Loire, pour des conditions de jusant de vive-eau en conjonction avec des débits fluviaux d'étiage, moyen et de crue. Compte tenu des observations, on peut estimer une valeur $\tau_{o}=2,50 \mathrm{~N} \mathrm{~m}^{-2}$ comme étant nécessaire pour remettre en suspension les couches de crème de vase. On peut noter que cette valeur de $\tau_{o}$ correspond au début d'érosion des vases de Loire de concentration inférieure à $320 \mathrm{~kg} \mathrm{~m}^{-3}$ (Sanchez et al., 2000) et concerne presque toute la couche de crème de vase.

Il semble que la Loire dispose actuellement d'une puissance suffisante pour entretenir les profondeurs en aval de Donges. On peut envisager à terme la mise en œuvre de travaux permettant de concentrer le courant de jusant le long de la rive nord en aval de Donges. Cela consiste à réduire la section du chenal en jusant, ce qui revient à mieux ajuster les points de la figure 3 en alignant les points "aval" avec les points "amont", qui correspondent à des sections auto-draguées dans les conditions actuelles. La figure 5 montre une esquisse de solution possible. Cette solution est compatible avec deux alternatives d'aménagement récemment étudiées :

a) Un développement portuaire dans l'île de Bilho (projet appelé «fleuve port »), b) Une construction d'un ouvrage de déconnexion entre l'aval et l'amont de l'estuaire (qui pourrait se limiter au sud de l'île de Bilho).

Dans la solution envisagée, un ouvrage de déconnexion entre l'aval et l'amont de l'estuaire se situerait aux environs de Paimboeuf. Cet ouvrage s'effacerait pendant le flot mais serait activé pendant le jusant.

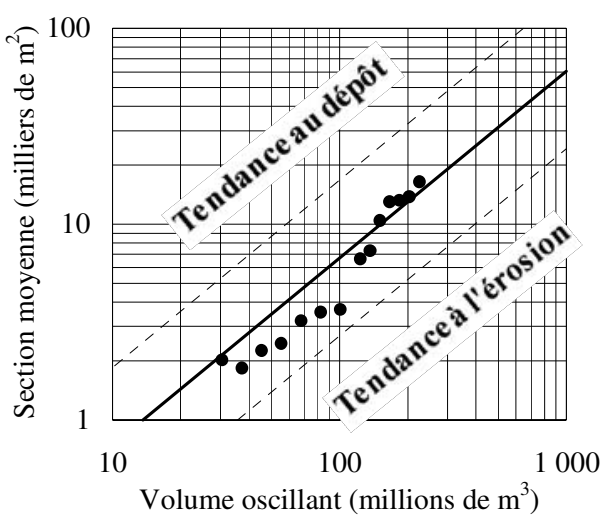

Figure 3 : Section moyenne en fonction du volume d'eau oscillant. En trait continu la loi de tendance générale. En traits discontinus les limites de la zone de stabilité dans $95 \%$ des cas.

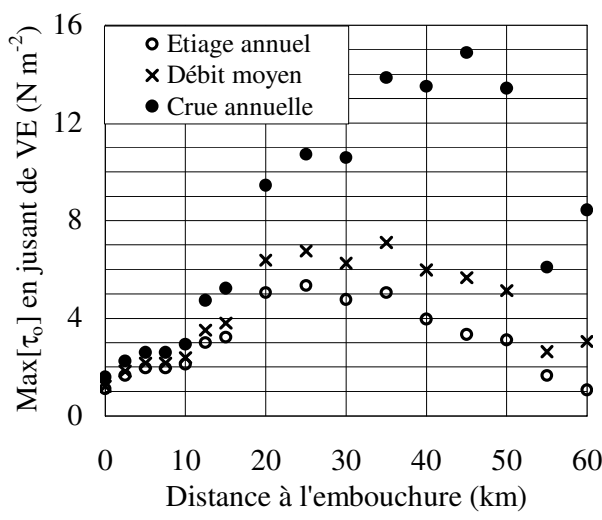

Figure 4 : Valeurs maximales de la contrainte de cisaillement au fond $\tau_{\mathrm{o}}$ en jusant de vive-eau dans l'estuaire de la Loire, calculées pour trois conditions différentes de débit fluvial.

\section{Aménagements légers pour la pêche et la plaisance dans l'estuaire}

Dans l'estuaire de la Loire on peut dénombrer plusieurs aménagements légers pour la pêche et pour la plaisance. Parmi ceux-ci, deux subissent l'envasement d'une façon très accentuée: le port-abri de Trentemoult dans l'agglomération nantaise, et le port du Dareau dans la ville de Couëron. 


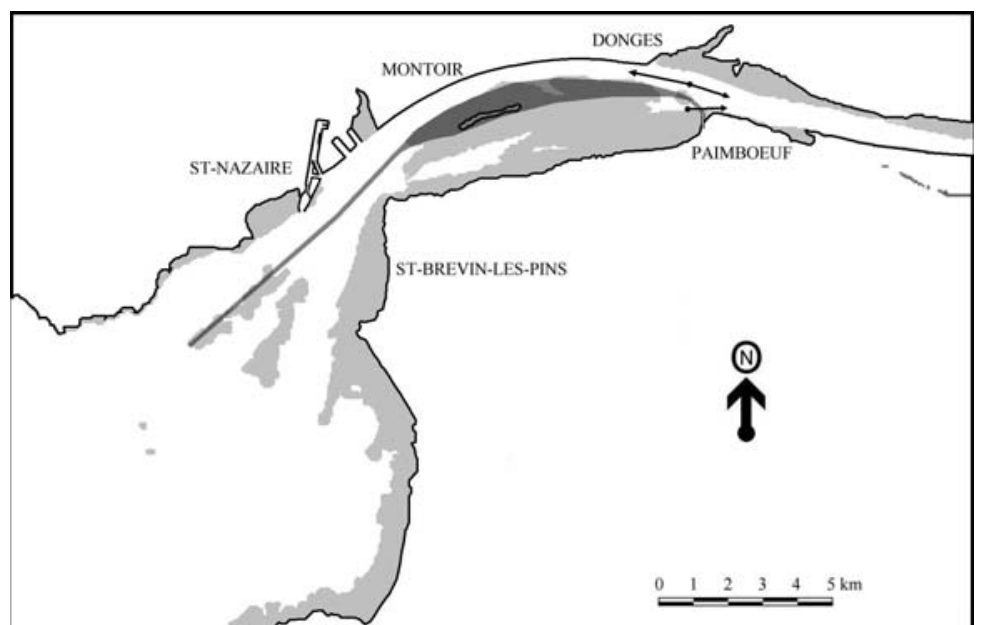

Figure 5 : Illustration d'un schéma d'aménagement possible permettant un curage des fonds du chenal aval de l'estuaire de la Loire par les courants de jusant.

\subsection{Le port-abri de Trentemoult}

Créé en 1979 suivant une étude effectuée en 1965, ce port est situé dans la commune de Rezé, sur la rive gauche de la Loire (figure 6). La superficie du bassin du port est de $8000 \mathrm{~m}^{2}$. Il peut accueillir environ 60 embarcations sur ses trois pontons pourvus de catways.

A chaque fois que des travaux de dragage ont été effectués, on a observé un envasement consécutif très rapide. En mai 1979 la cote du fond était de $-1,50 \mathrm{~m}$ CM. A partir de ces conditions initiales on a observé les envasements suivants : 2,30 m sur 1,5 mois, 2,70 m sur 4 mois, 3,20 m sur 8 mois.

Plusieurs autres campagnes de dragage ont été effectuées depuis 1979, avec un taux d'envasement d'autant plus élevé que la cote de dragage était plus basse. On constate que le fond devient stable à une cote voisine de $+3,50 \mathrm{~m} \mathrm{CM}$ (équilibre entre le dépôt et l'érosion provoquée par les petits courants, mais aussi par la pluie). Ces forts taux d'envasement s'expliqueraient d'une part par l'emplacement du port dans la zone du bouchon vaseux de l'estuaire, et d'autre part par sa situation d'anse en marge de la Loire, engendrant une zone de calme hydraulique.

\subsection{Le port de Couëron}

L'embouchure de l'étier du Dareau sur la rive droite de la Loire a servi pendant longtemps de lieu d'échouage pour des petites embarcations de pêche. Un aménagement de ce site a été mis en œuvre en 2002 (figure 7). On a notamment modifié la configuration des berges et mis en place un ponton principal pouvant recevoir environ 50 embarcations et un ponton supplémentaire pour les visiteurs.

Ces travaux ont induit un élargissement important de l'étier alors que le volume d'eau oscillant en amont du port est resté inchangé. Par la suite, on a observé un certain nombre de problèmes liés à l'envasement. Un niveau moyen d'équilibre semble être établi à une cote voisine de $+3,00 \mathrm{~m} \mathrm{CM}$. Tout dragage au dessous de ce niveau est inutile car un envasement ramène très rapidement le fond à cette cote. 


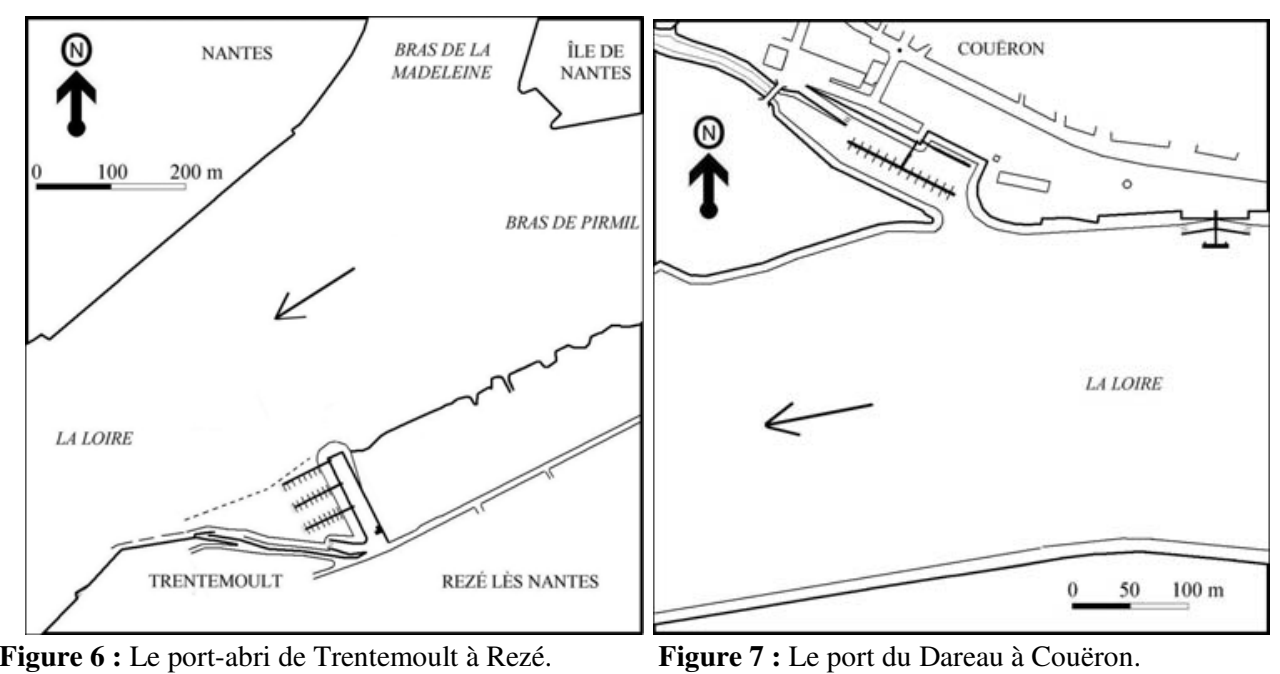

De plus, les cotes du fond sont très variables, ce qui provoque des déformations très importantes sur les pontons lorsque ceux-ci échouent. Ce phénomène est lié à la formation d'un chenal sinueux de petite largeur par lequel l'étier se vidange en basse mer. Au fond du chenal la cote est d'environ $+2,00 \mathrm{~m} \mathrm{CM}$, alors qu'en dehors du chenal on observe des monticules de vase pouvant avoir une hauteur d'environ $3 \mathrm{~m}$ par rapport à cette cote.

\subsection{Les type de port à privilégier dans la zone du bouchon vaseux}

Les cas des ports de plaisance de Trentemoult et de Couëron montrent la forte tendance à l'envasement des bassins à flot (ces deux ports ont été conçus avec des basins à flot avant de devenir des bassins d'échouage) aménagés en marge du cours d'eau dans la zone du bouchon vaseux de l'estuaire. En revanche, en dehors de la zone du bouchon vaseux, ce type d'aménagement ne pose pas de problème aigu de sédimentation, comme on peut le constater dans le site de la Roche Percée, situé $15 \mathrm{~km}$ en amont de Nantes sur la rive gauche.

Pour un bassin portuaire, la présence d'une porte, ou mieux, d'une écluse, semble indispensable pour limiter l'entrée des matières en suspension et pour bloquer à l'extérieur la crème de vase déplacée par les courant de densité. Un port avec écluse devrait être construit prochainement sur l'île de Nantes.

Une solution particulièrement adéquate dans ce secteur est le port à sec, compte tenu de la possibilité de compléter ces installations avec un ponton longeant le chenal, où l'entretien des profondeurs est assuré par le courant. On note que des pontons parallèles aux berges pouvant accueillir des bateaux de plaisance et de pêche sont implantés à Paimbœuf, Couëron, Nantes, et autres sites.

Enfin, les étiers et les canaux débouchant vers l'estuaire peuvent permettre un échouage à l'abri des forts courants. La section dans ces canaux dépendrait du volume d'eau oscillant selon le prolongement de la loi de stabilité présentée sur la figure 3. La profondeur peut donc augmenter si la largeur est restreinte localement. Le port de Cordemais donne un bon exemple d'un canal entretenu par 
les courants (lesquels sont engendrés par le rejet des eaux de refroidissement de la centrale thermique).

\section{Les ports de pêche et de plaisance du littoral}

Le taux d'envasement d'un port dépend d'une part de la turbidité des eaux environnantes, et d'autre part, de l'hydrodynamique locale associée à la configuration des ouvrages maritimes (digues, jetées, quais, pontons, seuils). En effet, d'une façon générale, l'implantation des ouvrages de protection des ports crée des conditions d'eau calme qui favorisent la sédimentation des matières en suspension et l'envasement des bassins.

Un paramètre simple pour évaluer les risques d'envasement est la masse solide en suspension, par unité de surface horizontale, entrant dans un port. Sachant que le marnage moyen en Loire-Atlantique est d'environ 3,55 m et qu'on compte 706 cycles de marée par an, la colonne d'eau équivalente est d'environ $2500 \mathrm{~m}$.

Pour estimer l'ordre de grandeur de l'envasement dans un bassin à flot, on peut faire l'hypothèse que $75 \%$ de la masse entrant dans le port se dépose à une concentration de $350 \mathrm{~kg} \mathrm{~m}^{-3}$. Trois ports du littoral sont présentés par la suite (figure 8).

a) Piriac-sur-mer est situé au nord-ouest du département, dans une zone où la turbidité moyenne de l'eau est de l'ordre de $0,020 \mathrm{~kg} \mathrm{~m}^{-3}$. Sachant que le port comporte une porte permettant de réduire de $25 \%$ le volume d'eau oscillant, on peut estimer l'envasement à $28 \mathrm{~kg} \mathrm{~m}^{-2} \mathrm{an}^{-1}$, soit $0,08 \mathrm{~m}$ par an. L'existence du seuil à l'entrée devrait encore limiter davantage l'entrée de sédiments en suspension près du fond.

b) La Gravette est située dans l'estuaire externe de la Loire, dans la commune de la Plaine sur mer. La turbidité moyenne de l'eau étant très forte, de l'ordre de $0,200 \mathrm{~kg} \mathrm{~m}^{-3}$, on évalue un envasement de $375 \mathrm{~kg} \mathrm{~m}^{-2} \mathrm{an}^{-1}$, soit $1,07 \mathrm{~m}$ par an.

c) Le Collet est situé dans la partie est de la baie de Bourgneuf, dans la commune des Moutiers. Même si la turbidité est très forte dans ce secteur (voir figure 2), l'envasement net observé est très faible, car grâce à la forme étroite du port, des chasses sont effectuées afin d'évacuer les matériaux déposés. Ce qui revient à dire que le taux net d'envasement dépend également de la forme du port et des possibilités de chasses à travers le bassin.

\section{Conclusions}

Les principaux résultats de cette étude sont les suivants :

a) Avec la configuration actuelle de la Loire, il apparaît qu'en descendant l'estuaire la contrainte tangentielle hydrodynamique au fond du chenal subit une forte réduction dans la section aval du fait de l'augmentation de la section transversale et de la profondeur. Pourtant il semble que la Loire dispose d'une énergie suffisante pour auto-draguer son chenal de navigation. La figure 5 illustre une solution possible permettant de concentrer le courant de jusant par 
la rive nord en aval de Donges afin de provoquer un important phénomène de chasse. Cependant, on doit nuancer cette solution et signaler que ce schéma d'aménagement constitue seulement une piste de réflexion visant à évacuer les vases fluides de l'estuaire. Des études plus approfondies seraient nécessaires pour démontrer son éventuelle efficacité et pouvoir ainsi comparer ses avantages et inconvénients avec ceux d'autres schémas d'aménagement envisagés actuellement dans l'estuaire aval.

b) La forte turbidité des eaux de l'estuaire en aval de Nantes entraîne un comblement très rapide de tout approfondissement effectué en marge de l'estuaire. Les solutions à préconiser dans ce secteur sont : échouage dans les étiers avec des aménagements sommaires, port à sec, pontons longeant le chenal de navigation et port à flot avec écluse.

c) Sur le littoral, le secteur où la turbidité est la plus faible se situe au nord du Croisic (figure 2). C'est dans ce secteur que l'envasement des ports est aussi le plus faible.

Une façon de limiter l'envasement dans les ports est l'implantation d'une porte, ou mieux, d'une écluse. Ceci afin de réduire le volume d'eau oscillant dans le port. Cette solution doit être examinée pour tout nouvel aménagement.

Dans tous les cas, les rejets en mer des vases de dragage sont conditionnés à une absence de pollution dans les sédiments. La collecte et le traitement des eaux résiduelles et de ruissellement sont donc nécessaires dans tous les ports.
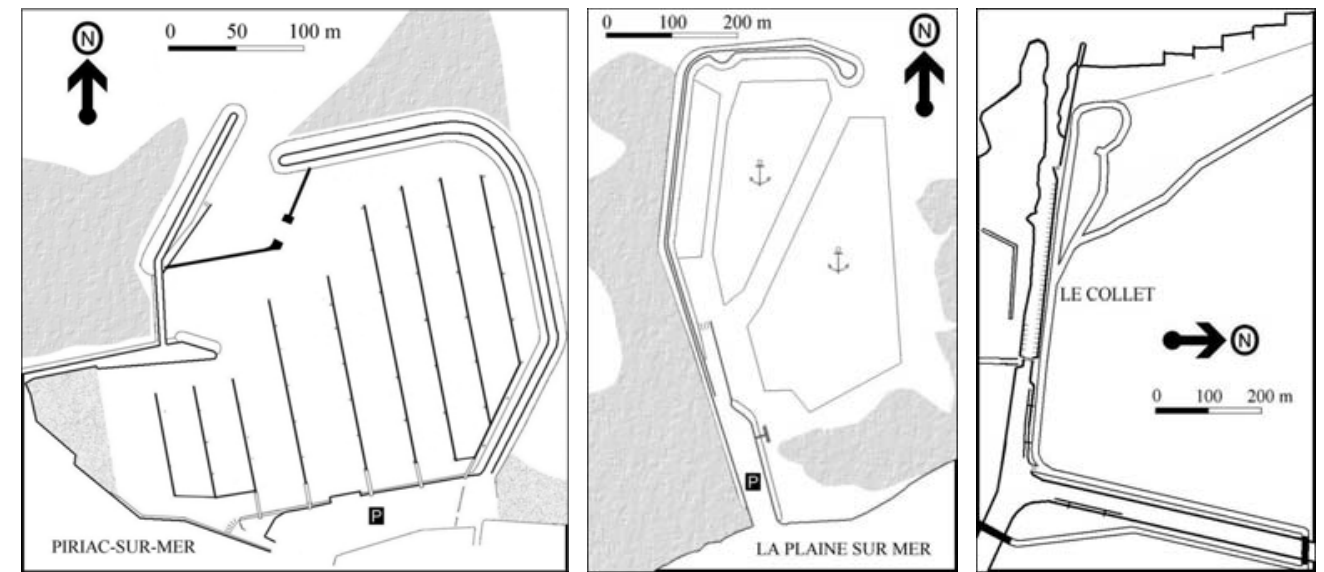

Figure 8 : Illustration de trois ports du littoral de Loire Atlantique. De gauche à droite : Piriac-sur-mer, La Gravette et Le Collet.

\section{Références bibliographiques}

Bowers, D.G., Ellis K.M., Jones S.E. (2005). Isolated turbidity maxima in shelf seas. Continental Shelf Research 25, 1071-1080.

Bruun, P. (1978). Stability of tidal inlets: theory and engineering. Elsevier, Amsterdam. 
Castaing, P. (1981). Le transfert à l'océan des suspensions estuariennes - Cas de la Gironde. Thèse de doctorat d'état ès sciences, Université Bordeaux I.

CMB Loire estuaire (2003). Rapport Etude Prospectives Aval. Programme interrégional Loire grandeur nature, Nantes

CSEEL (1984). Rapport final du comité scientifique pour l'environnement de l'estuaire de la Loire. Rapports Scientifiques et Techniques n ${ }^{\circ}$ 55, Publications du CNEXO, Brest.

Delft Hydraulics (1994). Etude hydrologique de l'estuaire de la Loire. Delft hydraulics, Delft, The Netherlands.

Denizeau, M. (1988). Nantes : les îles, le fleuve, le port. Documents d'histoire régionale, CRDP de Nantes.

DHI eau \& environnement (2003). Phénomènes sédimentaires estuariens : traitement et valorisation des données, Cellule de mesures et de bilans Loire estuaire, Nantes.

Fera P. 1994. Estuaire de la Loire. Agence de l'eau Loire Bretagne, Nantes.

Froidefond J.-M., Doxaran, D., Miller, P. (2003). Rapport final : acquisition et traitement d'images satellites. Programme interrégional Loire grandeur nature, Cellule de mesures et de bilans Loire estuaire, Nantes.

Gallenne B., (1974). Les accumulations turbides dans l'estuaire de la Loire - Etude de la "crème de vase". Thèse, Université de Nantes, 323 p.

Gouleau, D. (1975). Les premiers stades de la sédimentation sur les vasières littorales atlantiques - Rôle de l'émersion. Thèse de $3^{e}$ cycle, Université de Nantes.

Jarrett, J.T. (1976). Tidal prism-inlet area relationships, GITI Report 3, U.S. Army Engineer Waterways Experiment Station, Vicksburg, MS, USA.

LCHF (1987). Catalogue sédimentologique des côtes françaises - Côtes de la Manche et de l'Atlantique. Eyrolles, Paris.

Migniot, C. (1993). Bilan de l'hydrologie et de l'hydrosédimentaire de l'estuaire de la Loire au cours des deux dernières décennies. Association pour la protection de l'environnement de l'estuaire de la Loire - Port Autonome de Nantes - Saint Nazaire.

Migniot C., et Le Hir P. (1994). Estuaire de la Loire: Hydrosédimentaire. Rapports de synthèse de l'APEEL $\mathrm{n}^{\circ} 1$, Publication de l'association pour la protection de l'environnement de l'estuaire de la Loire, Nantes.

Papy, L. (1941). Les aspects naturels de la côte atlantique de la Loire à la Gironde. Introduction à une étude de géographie humaine. Delmas, Bordeaux.

Sanchez, M., Grovel, A., Hosseini, K. (2000). Impact sédimentaire des travaux d'aménagement de l'estuaire de la Loire. Bulletin of Engineering Geology and the Environment 59 (3), 239-246. 